\title{
No-reference image quality assessment based on regional mutual information
}

\author{
Yanjie Wang, Jianjun Zhou, Li Jia,Yue Wang \\ The Unit of 91635th, PLA, Beijing, China \\ Email: 2742069961@qq.com
}

\begin{abstract}
Keywords: Image quality assessment (IQA), no reference (NR), regional mutual information, wavelet transform, Support Vector Machine (SVM).

Abstract. We study the regional mutual information to build a powerful and effective no-reference (NR) image quality assessment (IQA) approach. This approach does not need to compute distortion-specific features, but use the features which can distinguish the distortion in images across the distortion types. Such features are extracted from the image regional mutual information, which includes modified regional mutual information and a novel way to describe such information via wavelet transform. Operating within the 2-step framework, these features are tested on the LIVE database and show a nice correlation with human subjective opinions of image quality.
\end{abstract}

\section{Introduction}

Nowadays, the photographs play a more and more important role in human social and daily life. For instance, Google takes billions of digital photographs to build its image search and that image search engine bring Google lots of application amount. In recent years, the massive sums of the digital images stay with the public and provide them the visual information. But the visual information maybe lost caused by image distortions, how to quantize the image distortion and visual information contained in image draw attention to the researchers.

Image quality assessment is the basic technology in image processing field. It can be classified into three approaches: Full-reference (FR), reduced-reference (RR) and no-reference (NR). Different approaches need different numbers of information from reference images. NR approach does not need the reference information, and this makes it to be a special concern. Recently, Saad et al. proposed holistic NR IQA algorithms referred to as BLIINDS-I and BLIINDS-II [1], [2]. Those algorithms extract the NSS features via a generalized Gaussian distribution (GGD) fit in the DCT domain of images using frequency coefficients. Moorthy et al. introduced the idea of a 2-stage framework for NR IQA, using NSS features of wavelet domain [3], [4]. Gao et al. using the heterogeneous property of multiple kernel learning with the NSS features to estimate image's quality [5]. Mittal et al. [6] utilized the normal and asymmetric generalized Gaussian distribution (AGGD) to fit the NSS features in luminance coefficients of the image and using SVM machine learning to build the mapping between features and image quality. But those algorithms do not consider the mutual information between the distorted image and itself, which is correlative with the image distortion. Here, we study the regional mutual information to build an effective and efficient no-reference image quality assessment approach.

The rest of the paper is organized as follows. In Sections II and III, we describe how to extract image features and predict image quality via those features and machine learning. We show the result of our algorithm in Section V and conclude the paper in Section VI.

\section{The Proposed Method}

Segmentation of image patches:Due to the theory that the image distortions do mainly affect the image quality in several image patches at the visual sensitive and insensitive places [7], the first step in our proceed is to divide the image in to patches whose size is $\mathrm{N} \times \mathrm{N}$. Those image patches cover the whole image but overlap adjacent others since the size of those patches are constant, but we do our best to make those overlap as small as possible. 
Given the collection of those patches, we just calculate a set of the regional mutual information (RMI) [8] coefficients of each patches. Furthermore, we consider the averaged RMI coefficients from each patch as the integral image RMI coefficients and the final image features.

Regional mutual information features: Having found many disadvantage exist in the previous mutual information, such as ignorance of spatial information of image, many researchers dedicate to develop a more robust mutual information calculation which is not based on the error assumption that the pixel and its neighboring pixels are independent [9]. One of the most effective approaches is the regional mutual information (RMI) [8]. In this paper, we utilize RMI method to measure the image distortion rate. Furthermore, to obtain RMI coefficients of the images more roundly, we calculate it from different factors, such as the size of the window, the step distance of the moving window and the orientation information. The details are listed as follows.

First, for each image patch extracting above, we calculated their RMI values. Here, instead of combining the reference image I0 and distortion image I in [9], we utilize the image intensity to estimate the matrix $\mathrm{p}$

$$
p=\text { window }_{r, o}(\mathrm{im})
$$

where $\mathrm{r}$ is the window size, and the o represents the motion orientation of the window, which includes the horizontal and vertical.

Second, we calculate the matrix $\mathrm{C}$ using $\mathrm{p}$ and the $\mathrm{RMI}$ is defined as follow:

$$
\begin{gathered}
C=\frac{1}{N} p * p^{T} \\
R M I(I)=H g\left(C_{A}\right)+H g\left(C_{B}\right)-H g(C)
\end{gathered}
$$

Where $H g(X)=\ln \left((2 \pi e)^{\frac{d}{2}} \operatorname{det}(X)^{\frac{1}{2}}\right)$, CA is the top left corner of the $\mathrm{C}$ with the $d / 2 \times d / 2$ size while the $\mathrm{CB}$ is the bottom right one of the $\mathrm{C}$ with the same size while the value $\mathrm{d}$ between 1 and $\mathrm{r}$.

At last, we totally calculate 12 sets of RMI value via different orientation o and window size $\mathrm{r}$. We also show one set of RMI in Fig. 1, and find that it is formed as considerable shock and multimodality. Therefore, it is unwise to fit it via any given function, such as Gaussian function, so we decide to utilize the mean value and the variance to describe the RMI values.

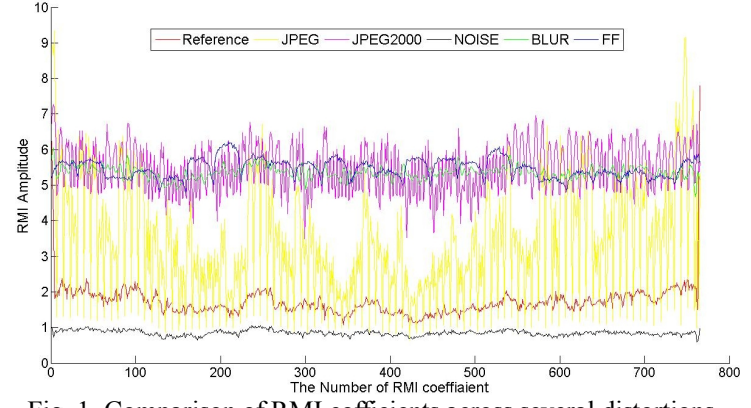

Fig. 1. Comparison of RMI cofficients across several distortions.

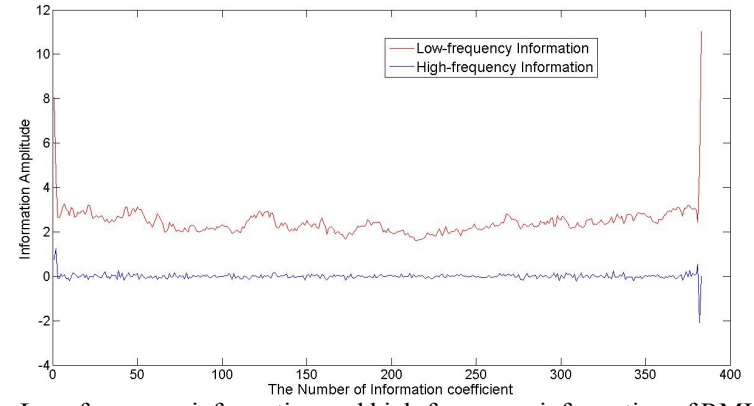

Fig. 2. Low-frequency information and high-frequency information of RMI

In order to obtain the mean value and the variance more accurately, we decompose the set of RMI values via wavelet transform to high-frequency and low-frequency information, respectively. The features contained the mean values of the low-frequency information and the variance from the high-frequency information (see Fig. 2).

The multiscale characteristic is the nature of the images, and the image distortions also affect the image quality via several scales. Furthermore, the multiscale characteristic has been utilized widely in image quality assessment application [10] [11], which can enhance the performance of those algorithms correlating with human perception. In this paper, we totally extract 48-dimension features which are constituted by 12-dimension features in two scales and two orientations. 


\section{Learning image quality evaluation}

To map extracted features to the image quality, we utilized the SVR model under the 2-step framework to evaluate the image quality. The 2-step framework has been wildly used in image quality assessment. The first step in this framework is that classify the image into specific distortion types and get the probabilities $\overrightarrow{\mathrm{P}}$, with which the image distortion type belongs to those specific types, using the SVM model trained by overall image. Then, assess the predicted image qualities $\vec{Q}$ in those specific distortion types utilizing the five SVR model trained by the image from each distortion type. At last, combine the probabilities $\overrightarrow{\mathrm{P}}$ and the image qualities $\overrightarrow{\mathrm{Q}}$ to get the final image quality Qf.

$$
Q_{f}=\dot{Q} \times \stackrel{\prime}{P}
$$

\section{Experiments and results}

The LIVE IQA database [12] is considered as the test database in our approach. In this database, there are 29 reference images and 779 corresponding distorted images which contain several types of image distortion, including white Gaussian noise, Gaussian blur, JPEG compression, JPEG2000 compression, and the Rayleigh fading channel which we usually called it fast fading distortion. Furthermore, the associated human differential mean opinion scores (DMOS) of each image, which represent the perceived quality of the image.

Considering feature learning, we divide the overall database into training set and testing set, using the training set to train the classification and regression models in 2-step framework and the testing set to testify the effectiveness of our approach. We randomly set the training set and testing set as the $80 \%$ of the reference image and associated distorted versions and $20 \%$ of the reference image and associated distorted versions, which were absolutely separated by content. Furthermore, we test our approach in the test measure described above across 1000 times, and the median performance evaluation indices across the 1000 iterations were used as the final algorithm performance evaluation.

The final indices for the performance of our approach are the Spearman's Rank Ordered Correlation Coefficient (SROCC), the linear correlation coefficient (LCC) and the Root Mean Squared Error (RMSE). Those indices are calculated using the predicted DMOS out from our approach and the real DMOS in database. It means the performance is better that the value of SROCC and LCC are more closed to 1 while RMSE value closes to 0 .

\section{Performance on LIVE Database}

Indicated by the indices SROCC, LCC and RMSE, the performance of our approach and other IQA approaches, including three full-reference approaches (PSNR, SSIM [13], [11] and VIF [14]) and four other holistic no-reference approaches (BIQI [4], DIIVINE [3], BLIINDS-II [1] and BRISQUE [6]), have been compared and listed in Tables I, II and III. With a similar random $20 \%$ testing set selection 1000 times, those FR and NR algorithms are considered as the same. We also recall all the performance indicators from 1000 trials and then a box plot of the SROCC distribution for each algorithm is shown in Fig.3. We can find from the results that our approach has a well correlation with the DMOS of test images, and its performance is highly competitive with other NR methods.

Table 1. Median SROCC across 1000 train-test trials on LIVE IQA database

\begin{tabular}{l||c|c|c|c|c|c}
\hline & JP2K & JPEG & NOISE & BLUR & FF & ALL \\
\hline PSNR & 0.8990 & 0.8484 & 0.9835 & 0.8076 & 0.8986 & 0.8293 \\
\hline SSIM & 0.9510 & 0.9173 & 0.9697 & 0.9513 & 0.9555 & 0.8996 \\
\hline VIF & 0.9515 & 0.9104 & 0.9844 & 0.9722 & 0.9631 & 0.9521 \\
\hline BIQI & 0.8551 & 0.7767 & 0.9764 & 0.9258 & 0.7695 & 0.7599 \\
\hline DIIVINE & 0.9352 & 0.8921 & 0.9828 & 0.9551 & 0.9096 & 0.9174 \\
\hline BLIINDS-II & 0.9462 & 0.9350 & 0.9634 & 0.9336 & 0.8992 & 0.9331 \\
\hline BRISQUE & 0.9442 & 0.9213 & 0.9891 & 0.9534 & 0.9042 & 0.9429 \\
\hline Ours & 0.9316 & 0.9550 & 0.9734 & 0.9617 & 0.8871 & 0.9423 \\
\hline
\end{tabular}


Table 2. Median LCC across 1000train-test trials on the LIVE database

\begin{tabular}{l||c|c|c|c|c|c}
\hline & JP2K & JPEG & NOISE & BLUR & FF & ALL \\
\hline PSNR & 0.8837 & 0.8515 & 0.9817 & 0.8006 & 0.8939 & 0.8081 \\
\hline SSIM & 0.9601 & 0.9485 & 0.9861 & 0.9537 & 0.9616 & 0.9100 \\
\hline VIF & 0.9664 & 0.9478 & 0.9924 & 0.9774 & 0.9698 & 0.9520 \\
\hline BIQI & 0.8414 & 0.7603 & 0.9732 & 0.9118 & 0.7342 & 0.7422 \\
\hline DIIVINE & 0.9409 & 0.9097 & 0.9744 & 0.9393 & 0.9128 & 0.9116 \\
\hline BLIINDS-II & 0.9493 & 0.9505 & 0.9614 & 0.9375 & 0.9079 & 0.9241 \\
\hline BRISQUE & 0.9499 & 0.9280 & 0.9887 & 0.9497 & 0.9176 & 0.9330 \\
\hline Ours & 0.9611 & 0.9722 & 0.9780 & 0.9626 & 0.9070 & 0.9508 \\
\hline
\end{tabular}

Table 3.Median RMSE across 1000train-test trials on LIVE database

\begin{tabular}{l||c|c|c|c|c|c}
\hline & JP2K & JPEG & NOISE & BLUR & FF & ALL \\
\hline PSNR & 7.5641 & 8.3269 & 3.0741 & 9.4289 & 7.3990 & 9.4973 \\
\hline SSIM & 4.5389 & 5.0771 & 2.6584 & 4.6823 & 4.4855 & 6.6355 \\
\hline VIF & 4.1943 & 5.0856 & 1.9608 & 3.3315 & 3.9624 & 4.9180 \\
\hline BIQI & 13.787 & 17.013 & 5.3804 & 9.6562 & 15.551 & 15.954 \\
\hline DIIVINE & 8.5703 & 10.607 & 5.2137 & 8.0663 & 9.6520 & 9.9347 \\
\hline BLIINDS-II & 8.1730 & 7.7658 & 6.5009 & 8.0696 & 9.7141 & 9.0473 \\
\hline BRISQUE & 8.0293 & 9.3685 & 3.4819 & 7.4852 & 9.2958 & 8.7074 \\
\hline Ours & 7.9862 & 8.2415 & 6.8919 & 6.4349 & 12.946 & 9.7058 \\
\hline
\end{tabular}

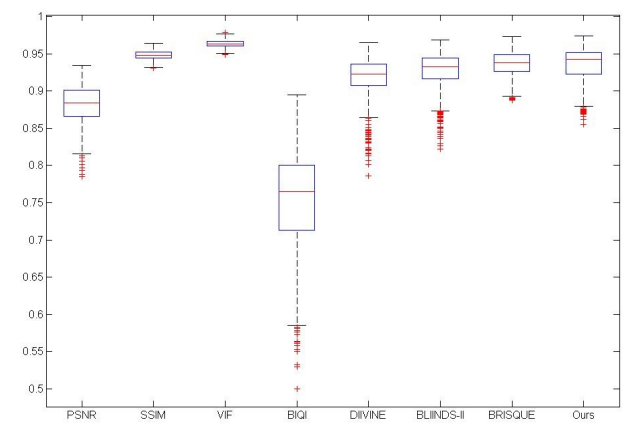

Fig. 3. Box plot of SROCC distributions of IQA models across 1000 train-test trials on the LIVE IQA database.

\section{Classification Analysis}

Due to the first step that classified the test image into different distortion types in 2-step framework, we also show the classification performance of our approach in Table IV. Similarly, we compare the classification using testing and training set across all distortion categories over the 1000 trials, and utilize the medians of the classification accuracies as final indices. There the hard classification is not applied in the 2-stage framework used in our approach but a weighted probability estimate.

\section{Conclusion}

In this paper, we presented a new NR IQA approach which uses a novel way to evaluate the distorted image quality disregarding the distortion types. The major contributions in our research are described as three points. First, we expand the application of RMI into the NR image quality assessment, which has been proved that it has a nice performance in FR image quality assessment [6]. Second, the images are segmented into image patches in order to catch the image distortion more effectively. Finally, we build an effective way to describe the irregular distribution, using the wavelet decompose the high- and low- frequency and calculating the mean and variance values as the element for description.

In the future, we will make further efforts to improve this approach, such as expending it into NR-IQA model without feature learning procedure. 


\section{References}

[1] M. A. Saad, A. C. Bovik, and C. Charrier, "Blind image quality assessment: a natural scene statistics approach in the DCT domain," IEEE Trans. Image Process., vol. 21, no. 8, pp. 3339-3352, Aug. 2012.

[2] M. A. Saad, A. C. Bovik, and C. Charrier, "A DCT statistics-based blind image quality index," IEEE Signal Process. Lett., vol. 17, no. 6, pp. 583-586, Jun. 2010.

[3] A. K. Moorthy and A. C. Bovik, "Blind image quality assessment: From natural scene statistics to perceptual quality," IEEE Trans. Image Process., vol. 20, no. 12, pp. 3350-3364, Dec. 2011.

[4] A. K. Moorthy and A. C. Bovik, "A two-step framework for constructing blind image quality indices," IEEE Signal Process. Lett., vol. 17, no. 5, pp. 513-516, May. 2010.

[5] X. Gao, F. Gao, D. Tao, and X. Li, "Universal Blind Image Quality Assessment Metrics Via Natural Scene Statistics and Multiple Kernel Learning," IEEE Trans. Neural Networks and Learning Systems, vol. 24, no. 12, pp. 2013-2026, Dec. 2013.

[6] A. Mittal, A. K. Moorthy and A. C. Bovik, "No-reference image quality assessment in the spatial domain,” IEEE Trans. Image Process., vol. 21, no. 12, pp. 4695-4708, Dec. 2012.

[7] A. Mittal, R. Soundararajan and A. C. Bovik, "Making a 'Completely Blind' Image Quality Analyzer," IEEE Signal Processing Letters, vol. 20, no. 3, pp. 209-212, Mar. 2013.

[8] B. Daniel Russakoff, C. Tomasi, T. Rohlfing and C. R.Maurer, "Image similarity using mutual information of regions", Computer Vision-ECCV, vol.3023, pp.596-607, 2004.

[9] J. Li, X. Zhang and M. Ding, "Image quality assessment based on regional mutual information", 2011 International Conference on Intelligent Computation and Bio-Medical Instrumentation

[10] L. He, D. Tao, X. Li, and X. Gao, "Sparse representation for blind image quality assessment," IEEE Conference on Computer Vision and Pattern Recognition, pp. 1146-1153, Jun. 2012.

[11] Z. Wang, A. C. Bovik, H. R. Sheikh and E. P. Simoncell, "The SSIM index for image quality assessment," online at HUhttp://www.cns.nyu.edu/ lcv/ssimU

[12] H. R. Sheikh, Z. Wang, L. Cormack and A. C. Bovik, "LIVE Image Quality Assessment Database Release 2", http://live.ece.utexas.edu/research/quality.

[13] Z. Wang, A. C. Bovik, H. R. Sheikh and E. P. Simoncell, "Image quality assessment: from error visibility to structural similarity,” IEEE Trans. Image Process., vol. 13, no. 4, pp. 600-612, Apr. 2004.

[14] H. R. Sheikh and A. C. Bovik, "Image information and visual quality," IEEE Trans. Image Process., vol. 15, no. 2, pp. 430-444, Feb. 2006. 